\title{
Integrable systems and Special Kähler metrics
}

\author{
Nigel Hitchin \\ Mathematical Institute, Woodstock Road, Oxford, OX2 6GG \\ hitchin@maths.ox.ac.uk
}

October 14, 2019

\section{Introduction}

The base of an algebraic completely integrable system has a natural differential geometric structure called a Special Kähler structure, describing in a rather different way the variation of the period matrix of the abelian varieties which form the fibres. The moduli space $\mathcal{M}$ of Higgs bundles on a curve $\Sigma$ of genus $g>1$ gives a particularly interesting example - the so-called Hitchin system. In [2] the authors give a simple formula for the Kähler potential of the Special Kähler structure in this case. Here we give first a short survey of this situation and afterwards extend it to cover some distinguished integrable subsystems.

A Higgs bundle is a pair $(V, \Phi)$ consisting of a holomorphic vector bundle $V$ of rank $n$ and a holomorphic section (the Higgs field) of End $V \otimes K$, where $K$ is the canonical bundle, satisfying a joint stability condition. The integrable system arises from taking the curve $S$ defined by $\operatorname{det}(x-\Phi)=x^{n}+a_{1} x^{n-1}+\cdots+a_{n}=0$, and realizing $(V, \Phi)$ by the direct image construction from a line bundle on $S$. The spectral curve sits naturally in the total space of the cotangent bundle $T^{*} \Sigma$ and the formula in [2] is

$$
K=\operatorname{Im} \frac{1}{4} \int_{S} \theta \wedge \bar{\theta}
$$

where $\theta$ is the canonical one-form on the cotangent bundle.

The assumption here is that $S$ is smooth. However, the formula makes sense for a singular spectral curve and our aim is to show that indeed it holds on the subintegrable systems which come from fixing the singularity type - here we restrict to ordinary 
singularities of $S$, points of multiplicity $m$ with $m$ distinct tangents. For a smooth curve $S$ the fibre of the integrable system is its Jacobian. For a singular curve it is the Jacobian of the normalization.

We end with a discussion of examples and links to the asymptotics of the natural hyperkähler metric on the moduli space $\mathcal{M}$.

\section{$2 \quad$ Integrable systems}

\subsection{The geometry of integrable systems}

By an integrable system we mean a symplectic manifold $(M, \omega)$ of dimension $2 n$ and a proper map $h: M \rightarrow B$ to an $n$-dimensional manifold $B$ with Lagrangian fibres. The connected component of the fibre over a regular value is a torus, and functions on $B$ composed with $h$ Poisson commute. For the moment we work over the real numbers.

A tangent vector $X \in T_{a} B$ lifts canonically to a section of the normal bundle of the fibre $M_{a}$ - the first order deformation of $M_{a} \subset M$ - and locally we can extend this to a vector field $\tilde{X}$ along $M_{a}$, well-defined up to the addition of a vector field $Y$ tangent to $M_{a}$. But $M_{a}$ is Lagrangian, so the interior product $i_{Y} \omega$ restricts to zero and therefore $i_{\tilde{X}} \omega$ is a well-defined global 1-form on the fibre. It is in fact a closed 1-form. The de Rham cohomology class $\left[i_{\tilde{X}} \omega\right]$ now provides a natural isomorphism $T_{a} B \cong H^{1}\left(M_{a}, \mathbf{R}\right)$.

If $U \subset B$ is a contractible neighbourhood of $a$ then homotopy invariance means the restriction map $H^{1}\left(h^{-1}(U), \mathbf{R}\right) \rightarrow H^{1}\left(M_{a}, \mathbf{R}\right)$ is an isomorphism. If $a, b \in U$ then this gives a natural isomorphism $T_{a} B \cong T_{b} B$. Globally this is a flat connection on the tangent bundle of $B$. Since $\omega$ vanishes on $M_{a}$ its cohomology class in $H^{2}\left(h^{-1}(U), \mathbf{R}\right)$ is zero and there we can write $\omega=d \theta$. Choosing a basis $C_{i} \in H_{1}\left(M_{a}, \mathbf{Z}\right) \cong \mathbf{Z}^{n}$ one obtains local flat coordinates

$$
x_{i}=\int_{C_{i}} \theta
$$

It follows that the connection $\nabla$ is flat and torsion-free.

Now suppose that $M$ is a holomorphic symplectic manifold and $h: M \rightarrow B$ is holomorphic. Then the real and imaginary parts of the holomorphic symplectic form $\omega^{c}$ are real symplectic forms. Let $\omega$ be the real part. Suppose in addition that $M$ has a Kähler form $\Omega$. Then the cohomology class $[\Omega] \in H^{2}(M, \mathbf{R})$ defines a real 
symplectic form on the base:

$$
(X, Y)=\int_{M_{a}} i_{\tilde{X}} \omega \wedge i_{\tilde{Y}} \omega \wedge \Omega^{n-1} .
$$

This is just the cup product of cohomology classes and so is constant with respect to the flat connection. Hence the base $B$ is equipped with a flat symplectic connection.

The complex structure on the base is not flat. It is an endomorphism $I: T \rightarrow T$ such that $I^{2}=-1$ or equivalently a section of $T^{*} \otimes T$. This is a 1 -form with values in the flat vector bundle $T$ and the condition it satisfies is $d_{\nabla} I=0$ where $d_{\nabla}$ is the flat de Rham differential operator $d_{\nabla}: \Omega^{1}(B, T) \rightarrow \Omega^{2}(B, T)$. Locally $I=d_{\nabla} X$ for a Hamiltonian vector field.

Remark: The above data - a flat torsion-free symplectic connection and a complex structure $I$, compatible with the symplectic form and satisfying $d_{\nabla} I=0$ - is the definition of a Special Kähler metric on $B$ in the approach of D.Freed [6], directed towards mathematicians. The original concept appeared in the physics literature in the context of supersymmetry [12, [7].

We could have chosen the imaginary part of $\omega^{c}$ or the real part of $e^{i \theta} \omega^{c}$ to get other flat connections and this is all part of the rich geometry of Special Kähler stuctures but we shall restrict ourselves here to one aspect, which is a convenient description of the metric given in [6] in terms of a Kähler potential.

In the holomorphic situation the fibre $M_{a}$ is a complex torus and the Kähler class of $\Omega$ means we can choose a symplectic basis $A_{i}, B_{i}$ of $H_{1}\left(M_{a}, \mathbf{R}\right)$. Let $x_{i}, y_{i}, 1 \leq i \leq n$ be the real flat coordinates corresponding to this basis, then they are the real parts of holomorphic coordinates $z_{i}$ and $w_{i}$ and a Kähler potential is given by

$$
K=\frac{1}{2} \operatorname{Im} \sum_{i} w_{i} \bar{z}_{i} .
$$

If $\omega^{c}=d \theta^{c}$ and $\alpha_{i}, \beta_{i}$ denote the dual basis of $H^{1}\left(M_{x}, \mathbf{R}\right)$ then $\left[\theta^{c}\right]=\sum_{i} z_{i} \alpha_{i}+w_{i} \beta_{i}$ and the above formula can be written as

$$
K=\operatorname{Im} \int_{M_{a}} \theta^{c} \wedge \bar{\theta}^{c} \wedge \Omega^{n-1} .
$$

\subsection{Higgs bundles}

The particular holomorphic integrable system we want to apply this formula to is where $M=\mathcal{M}$ is the moduli space of Higgs bundles on a Riemann surface $\Sigma$ of genus 
$g>1$. A Higgs bundle is a rank $n$ vector bundle $V$ together with a holomorphic section $\Phi$ of End $V \otimes K$ where $K$ is the canonical bundle. A stability condition for the pair gives rise to a well-behaved moduli space which is a holomorphic symplectic manifold.

We can represent a Higgs bundle as a pair $(A, \Phi)$ where $A$ denotes a $\bar{\partial}$-operator $\bar{\partial}_{A}: \Omega^{0}(\Sigma, V) \rightarrow \Omega^{01}(\Sigma, V)$ on the rank $n$ holomorphic vector bundle $V$ and $\Phi$, the Higgs field, is a section of End $V \otimes K$ such that $\bar{\partial}_{A} \Phi=0$.

In this Dolbeault form a tangent vector to the moduli space $\mathcal{M}$ is given by a pair $(\dot{A}, \dot{\Phi})$ satisfying $\bar{\partial}_{A} \dot{\Phi}+[\dot{A}, \Phi]=0$ modulo the action of a $C^{\infty}$ endomorphism $\psi$ defining $(\dot{A}, \dot{\Phi})=\left(\bar{\partial}_{A} \psi,-[\psi, \Phi]\right)$. The symplectic form is then

$$
\omega^{c}\left(\left(\dot{A}_{1}, \dot{\Phi}_{1}\right),\left(\dot{A}_{2}, \dot{\Phi}_{2}\right)\right)=\int_{\Sigma} \operatorname{tr}\left(\dot{A}_{1} \dot{\Phi}_{2}-\dot{A}_{2} \dot{\Phi}_{1}\right)
$$

The characteristic polynomial of $\Phi$ gives

$$
\operatorname{det}(x-\Phi)=x^{n}+a_{1} x^{n-1}+\cdots+a_{n}
$$

where the coefficient $a_{m}$ is a holomorphic section of $K^{m}$. Since the characteristic polynomial is conjugation-invariant these coefficients give a well-defined map

$$
h: \mathcal{M} \rightarrow \bigoplus_{i=1}^{n} H^{0}\left(\Sigma, K^{i}\right)
$$

This defines the integrable system. Later it will be useful to use the invariant polynomials $\operatorname{tr}\left(\Phi^{m}\right)$ instead of the coefficients $a_{i}$ to define an equivalent map $h$.

A point $a=\left(a_{1}, \ldots, a_{n}\right)$ in the base $\mathcal{B}$ defines an algebraic curve $\operatorname{det}(x-\Phi)=0$, the spectral curve $S$. This is an $n$-fold covering $\pi: S \rightarrow \Sigma$ and a line bundle $L$ on $S$ defines a rank $n$ bundle $V$ on $\Sigma$ by the direct image $\pi_{*} L$ : over an open set $U \subset \Sigma$, $H^{0}(U, V)=H^{0}\left(U, \pi_{*} L\right)=H^{0}\left(\pi^{-1}(U), L\right)$ by definition. The indeterminate $x$ in the characteristic polynomial is a section of $\pi^{*} K$ on $S$ and then $x: H^{0}\left(\pi^{-1}(U), L\right) \rightarrow$ $H^{0}\left(\pi^{-1}(U), L \pi^{*} K\right)$ defines a Higgs field $\Phi$ on $V$. In this way we can identify the fibre over a point $a$ which defines a smooth spectral curve (a regular value of $h$ ) with the Picard variety $\operatorname{Pic}^{d}(S)$ for a fixed $d$ depending on the degree of $V$. This is a torsor for the Jacobian of $S$ and is a complex torus.

The family of $\bar{\partial}$-operators $\bar{\partial}_{A}$ defines a determinant line bundle whose cohomology class is given by a Kähler (actually hyperkähler) form $\Omega$ and restricts to the theta class on the Jacobian [3]. So we have all the data for a Special Kähler metric on the base $\mathcal{B}$. 
We have one more feature which is the $\mathbf{C}^{*}$-action defined by $(V, \Phi) \mapsto(V, \lambda \Phi)$. From (2) it acts on the symplectic form $\omega^{c}$ by rescaling and so if $X$ is the holomorphic vector field on $\mathcal{M}$ generated by the infinitesimal action we have $\mathcal{L}_{X} \omega^{c}=\omega^{c}$ or $d\left(i_{X} \omega^{c}\right)+$ $i_{X} d \omega^{c}=\omega^{c}$ which, since $\omega^{c}$ is closed, means that $\omega^{c}=d\left(i_{X} \omega^{c}\right)$. Hence from (1) we have a formula for the Kähler potential of the Special Kähler metric

$$
K=\operatorname{Im} \int_{\mathcal{M}_{a}} i_{X} \omega^{c} \wedge \overline{i_{X} \omega^{c}} \wedge \Omega^{n-1} .
$$

Note the geometric interpretation of the 1 -form $i_{X} \omega^{c}$ on a fibre $\mathcal{M}_{a}$. If we regard it as a tangent vector on the base, or a first order deformation of the fibre, it is simply the deformation given by the action of $\mathbf{C}^{*}$.

\subsection{Spectral curves}

The base $\mathcal{B}$ of the integrable system now has two interpretations: the parameter space of Lagrangian fibres in $\mathcal{M}$ and the parameter space of spectral curves. Now, as shown in [8], a special Kähler structure exists on the moduli space of deformations of a compact complex Lagrangian in a complex symplectic Kähler manifold, and not just the fibres of an integrable system. The procedure is the same - a section of the normal bundle defines a holomorphic 1-form and its periods define the Special Kähler structure.

A special case consists of the spectral curves themselves. The equation $x^{n}+a_{1} x^{n-1}+$ $\cdots+a_{n}=0$ defines the zero set of a section of $\pi^{*} K^{n}$ on the total space $|K|$ of $K$ where $x$ is the tautological section of $\pi^{*} K$ on $|K|$. Thus $x$ embeds $S$ as a curve in the complex surface $|K|$, the cotangent bundle $T^{*} \Sigma$ of $\Sigma$, and the covering is given by the restriction of the projection $\pi: T^{*} \Sigma \rightarrow \Sigma$. The cotangent bundle is canonically symplectic and any curve is Lagrangian. Moreover, when $n=1$ the factor $\Omega^{n-1}$ disappears in the definition of the symplectic form on the moduli space, so we do not require a Kähler form on the surface.

All sections of $\pi^{*} K^{n}$ on $|K|$ are of the form $a_{0} x^{n}+a_{1} x^{n-1}+\cdots+a_{n}$ and compact zero sets require $a_{0} \neq 0$, thus any deformation is described by the equation of a spectral curve. In particular since the normal bundle to a curve in a symplectic surface is the canonical bundle $K_{S} \cong \pi^{*} K^{n}$.

In [2] Baraglia and Huang show that the two Special Kähler structures - the parameter space of fibres of $h: \mathcal{M} \rightarrow \mathcal{B}$ and of curves $S \subset T^{*} \Sigma-$ are the same. We now give a slightly different account of this result. 


\section{Two families of Lagrangians}

\subsection{Fibres of the integrable system}

Consider again the tangent space to the moduli space of Higgs bundles $\mathcal{M}$. As noted above this is defined by $(\dot{A}, \dot{\Phi})$ satisfying $\bar{\partial}_{A} \dot{\Phi}+[\dot{A}, \Phi]=0$ modulo $(\dot{A}, \dot{\Phi})=$ $\left(\bar{\partial}_{A} \psi,-[\psi, \Phi]\right)$. This space has a holomorphic interpretation as the hypercohomology $\mathbf{H}^{1}$ of the complex of sheaves:

$$
\mathcal{O}(\text { End } V) \stackrel{[\Phi,-]}{\rightarrow} \mathcal{O}(\text { End } V \otimes K)
$$

One of the two spectral sequences associated to this complex gives, using the vanishing theorem coming from stability, the following:

$$
0 \rightarrow H^{1}(\Sigma, \operatorname{ker} \Phi) \rightarrow \mathbf{H}^{1} \rightarrow H^{0}(\Sigma, \operatorname{coker} \Phi) \rightarrow 0
$$

Here ker $\Phi$ is the sheaf of centralizers of $\Phi$ and coker $\Phi$ the cokernel sheaf of the adjoint action of $\Phi$. Since $\bar{\partial}_{A} \dot{\Phi}=-[\dot{A}, \Phi]$ we see that $\dot{\Phi}$ is holomorphic modulo the image of ad $\Phi$ and so defines the image of $(\dot{A}, \dot{\Phi})$ in $H^{0}(\Sigma$, coker $\Phi)$.

If the spectral curve is smooth then $\Phi$ is everywhere regular and hence the centralizer is generated by $1, \Phi, \ldots, \Phi^{n-1}$ where $\Phi^{m}: K^{-m} \rightarrow$ End $V$. It follows that $\operatorname{ker} \Phi \cong$ $\mathcal{O} \oplus K^{-1} \oplus \cdots \oplus K^{-(n-1)}$ and similarly coker $\Phi \cong K \oplus K^{2} \oplus \cdots \oplus K^{n}$.

If $p: \mathcal{O}($ End $V) \rightarrow \operatorname{coker} \Phi$ is the projection then $\operatorname{tr}\left(\Phi^{m} \dot{\Phi}\right)=\operatorname{tr}\left(\Phi^{m} p(\dot{\Phi})\right)$ and then the right hand arrow in (3) is

$$
(\dot{A}, \dot{\Phi}) \mapsto\left(\operatorname{tr} \dot{\Phi}, \operatorname{tr}(\Phi \dot{\Phi}), \ldots, \operatorname{tr}\left(\Phi^{n-1} \dot{\Phi}\right)\right) \in \bigoplus_{m=1}^{n} H^{0}\left(\Sigma, K^{m}\right)
$$

We recognize this as the derivative of the projection $h: \mathcal{M} \rightarrow \mathcal{B}$ of the integrable system, and so the tangent space of a fibre is

$$
H^{1}(\Sigma, \operatorname{ker} \Phi) \cong \bigoplus_{m=0}^{n-1} H^{1}\left(\Sigma, K^{-m}\right)
$$

A tangent vector to the fibre is thus represented by

$$
\dot{A}=\sum_{m=0}^{n-1} \alpha_{m} \Phi^{m} \in \Omega^{01}(\Sigma, \text { End } V)
$$

where $\alpha_{m} \in \Omega^{01}\left(\Sigma, K^{-m}\right)$. 
The symplectic form $\omega^{c}$ on $\mathcal{M}$ gives a nondegenerate pairing between the tangent bundle of the fibre and base. In terms of the representatives above this is

$$
\int_{\Sigma} \operatorname{tr}(\dot{A} \dot{\Phi})=\sum_{m=0}^{n-1} \int_{\Sigma} \alpha_{m} \operatorname{tr}\left(\Phi^{m} \dot{\Phi}\right)=\sum_{m=0}^{n-1}\left\langle\left[\alpha_{m}\right], b_{m}\right\rangle
$$

where $\left(b_{1}, \ldots, b_{m}\right) \in H^{0}(\Sigma, K) \oplus \cdots \oplus H^{0}\left(\Sigma, K^{n}\right)$ is a tangent vector to the base and $\langle a, b\rangle$ is Serre duality on $\Sigma$.

The fibre is identified with the Jacobian of the spectral curve $S$ by the direct image. Recall that $x$ is a section of $\pi^{*} K$ and $\Phi=\pi_{*} x$, so $\dot{A}$ in (4) defines naturally

$$
\sum_{m=0}^{n-1} \pi^{*}\left[\alpha_{m}\right] x^{m} \in H^{1}(S, \mathcal{O})
$$

a tangent vector to the Jacobian. As noted, the canonical bundle of $S$ is isomorphic to $\pi^{*} K^{n}$ but we need to examine the isomorphism more closely to compare the Higgs bundle pairing above with the Serre duality pairing on the spectral curve $S$.

\subsection{Curves in $T^{*} \Sigma$}

The other family of Lagrangians are the compact curves $S \subset|K|$ in the linear system $\pi^{*} K^{n}$, each given by the vanishing of a section $s=x^{n}+a_{1} x^{n-1}+\cdots+a_{n}$. The surface $|K|=T^{*} \Sigma$ is the total space of the cotangent bundle of $\Sigma$, which has a canonical symplectic form $\omega$. If $z$ is a local coordinate on $\Sigma$ then $d z$ trivializes $K$ and $x \in \pi^{*} K$ is another local coordinate. The canonical 2-form is then $d x \wedge d z$.

The normal bundle $N$ of $S$ is $\pi^{*} K^{n}$ and the derivative of $s$ on $S$, assuming $S$ is smooth, is a nonvanishing section of $\pi^{*} K^{n} \otimes N^{*}$. Since the canonical bundle of $T^{*} \Sigma$ is trivial, $N \cong K_{S}$ and this gives an isomorphism $K_{S} \cong \pi^{*} K^{n}$. The function $z$ is a local coordinate on $S$ if $s_{x} \neq 0$ and locally the derivative of $s$ is $s_{x} d x+s_{z} d z$. Then the isomorphism is locally

$$
d z \mapsto s_{x}=n x^{n-1}+(n-1) a_{1} x^{n-2}+\cdots+a_{n-1} .
$$

The Abel-Jacobi map $S \rightarrow \operatorname{Jac}(S)$ realizes an isomorphism between holomorphic 1forms on $\Sigma$ and $S$ and also between $H^{1}(\Sigma, \mathbf{Z})$ and $H^{1}(\operatorname{Jac}(S), \mathbf{Z})$. The derivative of this map takes a tangent vector at a point $p \in S$ to a tangent vector to $\operatorname{Jac}(S)$. It comes from the long exact sequence of

$$
0 \rightarrow \mathcal{O}_{S} \rightarrow \mathcal{O}_{S}(p) \rightarrow \mathcal{O}_{p}(p) \rightarrow 0
$$


The derivative of the section of $\mathcal{O}(p)$ which vanishes at $p$ identifies the normal bundle of $p$ (i.e. the tangent space at $p$ ) with the line bundle $\mathcal{O}(p)$ so canonically $H^{0}\left(p, \mathcal{O}_{p}(p)\right) \cong T_{p}$ and the connecting homomorphism $\delta: T_{p} \cong H^{0}\left(p, \mathcal{O}_{p}(p)\right) \rightarrow$ $H^{1}(S, \mathcal{O}) \cong T \operatorname{Jac}(S)$ gives the corresponding tangent vector.

If $\pi(p)$ is not a branch point then $x$ takes $n$ distinct values $\lambda_{1}, \ldots, \lambda_{n}$ in a neighbourhood of $p$ so suppose $x=\lambda_{1}$ near $p$. Then define in a neighbourhood

$$
q(x)=\frac{\left(x-\lambda_{2}\right) \ldots\left(x-\lambda_{n}\right)}{\left(\lambda_{1}-\lambda_{2}\right) \ldots\left(\lambda_{1}-\lambda_{n}\right)}=c_{0}+c_{1} x+\cdots+c_{n-1} x^{n-1},
$$

equal to 1 when $x=\lambda_{1}$ and zero for $\lambda_{i}, i>1$. Note that the denominator is $s_{x}\left(\lambda_{1}\right)$, the identification of $\pi^{*} K^{n}$ with $K_{S}$. Applying $\delta$ gives a class in $H^{1}(S, \mathcal{O})$ of the form

$$
\sum_{m=0}^{n-1} \pi^{*} \delta\left(c_{m}\right) x^{m} \in H^{1}(S, \mathcal{O})
$$

where $\delta\left(c_{m}\right) \in H^{1}\left(\Sigma, K^{-m}\right)$.

Serre duality on $S$ pairs this localized class with a holomorphic 1-form by evaluation at $p$. Let $b_{i}$ be a holomorphic section of $K^{i}$, and consider $\pi^{*} b_{i} x^{n-i}$ a section of $\pi^{*} K^{n}$. Then $\pi^{*} b_{i} x^{n-i} q(x)$ modulo $\left(x-\lambda_{1}\right) \ldots\left(x-\lambda_{n}\right)$ is equal to $b_{i}(\pi(p)) \lambda_{1}^{n-i} / s_{x}\left(\lambda_{1}\right)$ at $p$ since $x^{n-i}-\lambda^{n-i}$ is divisible by $x-\lambda_{1}$.

On the other hand, the pairing via Higgs bundles in (5) is $c_{i} b_{i}(\pi(p))$ where $c_{i}=$ $(-1)^{n-i} \tau_{n-i} / s_{x}\left(\lambda_{1}\right)$ and $\tau_{k}$ is the $k$-th elementary symmetric function in $\lambda_{2}, \lambda_{3}, \ldots, \lambda_{n}$.

In terms of the full symmetric functions $\sigma_{i}$ we have $\tau_{k}=\sigma_{k}-\lambda_{1} \sigma_{k-1}+\lambda_{1}^{2} \sigma_{k-2}+\cdots+$ $(-1)^{k} \lambda_{1}^{k}$ so the homomorphism

$$
\left(b_{1}, b_{2}, \ldots, b_{n}\right) \mapsto \sum_{j=1}^{n} \pi^{*} b_{j}\left(x^{n-j}+\pi^{*} a_{1} x^{n-j-1}+\cdots+\pi^{*} a_{n-j}\right)
$$

identifies the tangent space of $\mathcal{B}$ with $H^{0}\left(S, \pi^{*} K^{n}\right) \cong H^{0}\left(S, K_{S}\right)$ in such a way that the Higgs bundle pairing is equal to the Serre duality pairing. This is Proposition 3.3 in [1].

\subsection{The Special Kähler metric}

Let $\mathcal{B}^{\text {reg }} \subset \mathcal{B}$ denote the space of smooth spectral curves, then we have identified the family of Jacobians with the Higgs bundle moduli space in a form where the pairing given by the symplectic forms coincides. The Abel-Jacobi map identifies holomorphic 
one-forms and their periods on the spectral curve with those of the Jacobian and it follows that the two Special Kähler metrics are the same.

Now the $\mathbf{C}^{*}$-action on $\mathcal{M}$ is given by $\Phi \mapsto \lambda \Phi$ and $\operatorname{det}(x-\lambda \Phi)=\lambda^{n} \operatorname{det}\left(\lambda^{-1} x-\Phi\right)$. So the action on spectral curves $S \subset T^{*} \Sigma$ is rescaling $x \mapsto \lambda^{-1} x$ of the fibre of the cotangent bundle. Call the associated vector field $Y$, then $i_{Y} \omega=\theta$ is the canonical 1-form on the cotangent bundle, and we have the following:

Proposition 1 ([2]) A Kähler potential for the Special Kähler metric on $\mathcal{B}^{\text {reg }}$ is given by

$$
K=\operatorname{Im} \frac{1}{4} \int_{S} \theta \wedge \bar{\theta}
$$

It is instructive to see the relationship between the two holomorphic 1-forms explicitly. In our local coordinates on $T^{*} \Sigma$ we have $\theta=x d z$. Using the identification of $K_{S}$ with $\pi^{*} K^{n}$ this becomes

$$
\begin{gathered}
x s_{x}=x\left(n x^{n-1}+(n-1) a_{1} x^{n-2}+\cdots+a_{n-1}\right)=n\left(-\left(a_{1} x^{n-1}+\cdots+a_{n}\right)\right)+(n-1) a_{1} x^{n-1}+\cdots \\
=-\left(a_{1} x^{n-1}+2 a_{2} x^{n-2}+\cdots+n a_{n}\right)
\end{gathered}
$$

using the equation of the spectral curve.

The $\mathbf{C}^{*}$-action on the Higgs field gives $\dot{\Phi}=\Phi$ and so $\operatorname{tr}\left(\Phi^{m} \dot{\Phi}\right)=\operatorname{tr} \Phi^{m+1}$ and we substitute $b_{j}=\operatorname{tr}\left(\Phi^{j}\right)$ in equation (6) . Newton's identity for sums of powers and elementary symmetric functions gives

$$
\sum_{j=1}^{k} b_{j} a_{k-j}=-k a_{k}
$$

and using this in (6) gives the above formula.

\section{Singular curves}

\subsection{Singular curves and singular fibres}

If $S \subset T^{*} \Sigma$ is now a singular curve then the formula of Proposition 1 still makes sense and a natural question is to understand the significance of the corresponding potential. When $S$ is singular, the fibre of the integrable system becomes singular. This means that there are points where some of the Hamiltonian vector fields corresponding to 
functions on the base vanish, or equivalently they are critical points of those functions. More geometrically, it is where the derivative of $h: \mathcal{M} \rightarrow \mathcal{B}$ is not surjective.

We observed that if $\Phi$ is regular everywhere then surjectivity holds so suppose $\Phi$ is regular except on a divisor $D \subset \Sigma$ (for example if the spectral curve $S$ is reduced), then the powers of $\Phi$ still lie in the centralizer but now there is a quotient sheaf $\mathcal{C}$ supported on $D$

$$
0 \rightarrow \mathcal{O} \oplus K^{-1} \oplus \cdots \oplus K^{-(n-1)} \rightarrow \operatorname{ker} \Phi \rightarrow \mathcal{C} \rightarrow 0
$$

From the long exact sequence

$$
\rightarrow H^{0}(D, \mathcal{C}) \stackrel{\delta}{\rightarrow} H^{1}\left(\Sigma, \mathcal{O} \oplus K^{-1} \oplus \cdots \oplus K^{-(n-1)}\right) \rightarrow
$$

sections of $\mathcal{C}$ define by Serre duality linear functions on the base of the integrable system $\mathcal{B}=H^{0}(\Sigma, K) \oplus \cdots \oplus H^{0}\left(\Sigma, K^{n}\right)$. These are functions which are critical at the point $(V, \Phi) \in \mathcal{M}$.

In rank 2, this critical locus was discussed in [9], with emphasis on a notion of nondegeneracy of the critical locus. Here we make the simplifying assumption that the singularities of $S$ are ordinary singularities in the classical terminology of Walker [11], namely that each singularity of multiplicity $m$ has $m$ distinct tangents. When $m=2$ this is a node. If $S$ has $k$ ordinary singularities of multiplicity $m_{j}$ and $\tilde{S}$ is the normalization of $S$ then its genus is

$$
g(\tilde{S})=g(S)-\sum_{1}^{k} \frac{m_{j}\left(m_{j}-1\right)}{2} .
$$

The fibres of the Higgs bundle integrable system are described in general by torsionfree sheaves on the spectral curve which can be regarded as the direct image of line bundles on a partial normalization. So consider the case of an ordinary singularity of $S$. At this point $m$ eigenvalues of $\Phi$ coincide. For simplicity assume that these are zero and $z$ is a local coordinate on $\Sigma$, then the equation of $S$ is locally of the form $\left(x-\lambda_{1} z\right)\left(x-\lambda_{2} z\right) \ldots\left(x-\lambda_{m} z\right)+$ higher order terms. Distinct tangents mean that the $\lambda_{i}$ are distinct.

The normalization of a neighbourhood of the singularity consists of $m$ disjoint discs $U_{i}$ on which $x=\lambda_{i} z+\cdots$ and so the direct image of $x$ contributes a component of the Higgs field of the form $z \phi+\cdots$ where $\phi$ is the diagonal matrix with entries $\lambda_{1}, \ldots, \lambda_{m}$. The quotient sheaf $\mathcal{C}$ above is thus generated by $\phi, \phi^{2}, \ldots, \phi^{m-1}$ modulo powers of $z \phi$ or $\sum_{j=1}^{m-1} p_{k}(z) \phi^{k}$ where $p_{k}(z)$ is a polynomial of degree $k-1$. Then at this singularity $\operatorname{dim} H^{0}(D, \mathcal{C})=1+2+\cdots+(m-1)=m(m-1) / 2$. 
Let $\mathcal{B}_{d} \subset \mathcal{B}$ denote the subvariety of spectral curves where $d$ denotes the singularity type ( $k$ ordinary singularities together with multiplicities $m_{k}$ ). Note that $\mathcal{B}_{d}$ is preserved by the $\mathbf{C}^{*}$-action. A tangent vector at a smooth point is annihilated by $\delta H^{0}(D, \mathcal{C})$ whose dimension is $\sum m_{k}\left(m_{k}-1\right) / 2$ and so the tangent space of $\mathcal{B}_{d}$ has dimension $g(\tilde{S})$ from (17). The generic fibre is isomorphic to the Jacobian of $\tilde{S}$ and so we have a subvariety of dimension $2 g(\tilde{S})$.

This is a symplectic submanifold and a subintegrable system $\mathcal{M}_{d}$, in fact the local structure can be realized as a symplectic quotient. If $\mathcal{B}_{d}$ is locally defined by functions $\left(f_{1}, f_{2}, \ldots, f_{m}\right)=0$, then these constitute the moment map for a $\mathbf{C}^{m}$-action which translates the torus fibres $\cong \operatorname{Jac}(\tilde{S})$. The symplectic quotient fibres over $\mathcal{B}_{d}$ and is locally holomorphically equivalent to the symplectic submanifold. As an integrable system in its own right, $\mathcal{B}_{d}$ inherits a Special Kähler structure and we shall determine next the Kähler potential by using the canonical 1-form $i_{X} \omega^{c}$.

\subsection{The subintegrable system}

The symplectic pairing on $\mathcal{M}_{d}$ provides an isomorphism between the tangent space of a fibre, namely $H^{1}(\tilde{S}, \mathcal{O})$, and the cotangent space of the fibre $\mathcal{B}_{d} \subset \mathcal{B}$. In particular there is an identification of the sections of $\pi^{*} K^{n}$ on $S$ which are annihilated by $\delta H^{0}(D, \mathcal{C})$ with holomorphic one-forms on $\tilde{S}$, the normalization of $S$. We need to see this concretely.

Near an $m$-fold singularity $x=0=z$, the section of $\pi^{*} K^{n}$ defining $S$ is of the form

$$
s=\left(x-\lambda_{1} z\right)\left(x-\lambda_{2} z\right) \ldots\left(x-\lambda_{m} z\right)+\cdots
$$

Then on the neighbourhood $U_{i} \subset \tilde{S}$ on which $x=\lambda_{1} z+\cdots$ we have

$$
s_{x}=z^{m-1} d x\left(\lambda_{1}-\lambda_{2}\right) \ldots\left(\lambda_{1}-\lambda_{m}\right)+\cdots
$$

and since the $\lambda_{i}$ are distinct this gives an isomorphism to $K_{\tilde{S}}$ after dividing by $z^{m-1}$. So if $p_{i} \in U_{i}$ are the points in the resolution of the singularity the derivative of $s$ gives an isomorphism $K_{\tilde{S}} \cong \pi^{*} K^{n}\left(-(m-1)\left(p_{1}+\cdots+p_{m}\right)\right)$. (This reduces the degree by $m(m-1)$ providing another argument for the genus $g(\tilde{S})$ whose degree is $2 g(\tilde{S})-2$.)

Now consider $\left(b_{1}, b_{2}, \ldots, b_{n}\right) \in H^{0}\left(\Sigma, K \oplus K^{2} \oplus \cdots \oplus K^{n}\right)$ annihilated by $\delta H^{0}(D, \mathcal{C})$. A class $\delta\left(p_{k}\right) \in H^{1}\left(\Sigma, K^{-k}\right)$ is represented by $c_{0}+c_{1} z+\ldots+c_{k} z^{k-1}$ modulo $z^{k}$ and if all such classes annihilate a section $b_{k}$ of $K^{k}$ then $b_{k}$ is divisible by $z^{k}$. Moreover since the equation of $S$ is $x^{n}+a_{1} x^{n-1}+\cdots=\left(x-\lambda_{1} z\right)\left(x-\lambda_{2} z\right) \cdots\left(x-\lambda_{m} z\right)+\cdots$, $a_{i}$ is divisible by $z^{i}$ if $i \leq m$. Finally, on the open set $U_{i}, x=\lambda_{i} z+$ higher order terms. Putting these three facts together, Equation [6] shows that the isomorphism 
from $\pi^{*} K^{n}$ to the canonical bundle given by the derivative of the section $s$ at smooth points of $S$ extends to the normalization $\tilde{S}$.

We can now follow the same procedure as in Sections 3.2 and 3.3 by choosing a generic point $p \in S$ to identify the periods of the form $i_{X} \omega^{c}$ on $\mathcal{M}$ restricted to $\mathcal{M}_{d}$ and the canonical one-form on $T^{*} \Sigma$ pulled back under the normalization map $p: \tilde{S} \rightarrow S \subset T^{*} \Sigma$.

To complete the picture of the Special Kähler metric we observe that the Kähler class on $\mathcal{M}$ was the first Chern class of the determinant line bundle. If we normalize the degree of $V$ to be $n(g-1)$ then there is a determinant divisor - the subvariety on which $H^{0}(\Sigma, V) \neq 0$. But if $V$ is the direct image of a line bundle $L$ on $\tilde{S}$ then $H^{0}(\Sigma, V)=H^{0}(\tilde{S}, L)$ by definition, so this class on the Jacobian is the class of the theta-divisor. The Special Kähler structure on $\mathcal{B}_{d}$ is therefore determined via the geometry of the normalization of the spectral curve. In particular, since $S$ and $\tilde{S}$ differ on a finite set of points, we obtain:

Proposition 2 A Kähler potential for the Special Kähler metric on $\mathcal{B}_{d}$ is given by

$$
K=\operatorname{Im} \frac{1}{4} \int_{S} \theta \wedge \bar{\theta}
$$

\section{$5 \quad$ Features and examples}

\subsection{Translation invariance}

Let $\alpha$ be a holomorphic 1-form on $\Sigma$ then it defines a diffeomorphism of $T^{*} \Sigma$ by translation in the fibre direction, mapping the spectral curve $x^{n}+a_{1} x^{n-1}+\cdots+a_{n}$ to $(x-\alpha)^{n}+a_{1}(x-\alpha)^{n-1}+\cdots+a_{n}$. The Kähler potential $K$ is now

$$
K=\operatorname{Im} \frac{1}{4} \int_{S}\left(\theta \wedge \bar{\theta}+\pi^{*} \alpha \wedge \bar{\theta}+\theta \wedge \pi^{*} \bar{\alpha}+\pi^{*} \alpha \wedge \pi^{*} \bar{\alpha}\right)
$$

This is the addition of $f+\bar{f}$ where $f$ is holomorphic on $\mathcal{B}$ and so leaves the metric unchanged. Thus $H^{0}(\Sigma, K)$ acts as an isometric group of translations. The quotient can be identified with the Special Kähler manifold for the moduli space of $\operatorname{PGL}(n, \mathbf{C})$ Higgs bundles. 


\subsection{Rank 2 bundles}

The lowest dimensional case for providing an example is when $V$ has rank 2 with $\Lambda^{2} V$ trivial and $\operatorname{tr} \Phi=0$ - these are $S L(2, \mathbf{C})$-Higgs bundles and the fibre of the integrable system is now the Prym variety inside the Jacobian of $S$. The spectral curve has a simple formula $x^{2}-q=0$ where $q \in H^{0}\left(\Sigma, K^{2}\right)$. In this case, since $x= \pm \sqrt{q}$, the formula for the Kähler potential can be written as an integral over $\Sigma$

$$
K=\frac{1}{2} \int_{\Sigma} \sqrt{q \bar{q}}
$$

Here $q \bar{q}$ is a section of $K^{2} \bar{K}^{2}$ so that $\sqrt{q \bar{q}}$ is a positive section of $K \bar{K}$ and therefore a measure. This is discussed also in [10]. The singularity type to determine $\mathcal{B}_{d}$ is now just a question of the multiple zeros of the quadratic differential $q$ and ordinary singularities are nodes.

Even simpler is the case where $g=2$ and $\Sigma$ is the hyperelliptic curve $y^{2}=f(z)=$ $\left(z-z_{1}\right) \ldots\left(z-z_{6}\right)$ for then a quadratic differential can be written as

$$
q=\left(c_{0}+c_{1} z+c_{2} z^{2}\right) \frac{d z^{2}}{f(z)}
$$

where $\left(c_{0}, c_{1}, c_{2}\right) \in \mathbf{C}^{3} \cong \mathcal{B}$. The Kähler potential is then an integral over the complex plane $\mathbf{C}$ :

$$
K=\frac{1}{2} \int_{\mathbf{C}} \frac{\left|c_{0}+c_{1} z+c_{2} z^{2}\right|}{|f(z)|} d z d \bar{z} .
$$

For $q$ to have one double zero requires $c_{0}+c_{1} z+c_{2} z^{2}$ to vanish at a branch point $z=z_{i}$. There are six of these and so six components of $\mathcal{B}_{1}$. The quadratic differential is $q=\left(a_{0}+a_{1} z\right)\left(z-z_{i}\right) d z^{2} / f(z)$ where $a_{0}+a_{1} z_{j} \neq 0$ ensures that there are no more singularities. Each component is thus the complement of six lines through the origin of $\mathbf{C}^{2}$.

Two double zeros implies $q=a\left(z-z_{i}\right)\left(z-z_{j}\right) d z^{2} / f(z)$ for $i \neq j$ so $\mathcal{B}_{2}$ has 15 components each one a copy of $\mathbf{C}^{*}$. Here the Kähler potential is $K=c|a|$ where $c$ is the constant

$$
c=\frac{1}{2} \int_{\mathbf{C}} \frac{\left|\left(z-z_{i}\right)\left(z-z_{j}\right)\right|}{|f(z)|} d z d \bar{z} .
$$

Setting $a=w^{2}$ this gives $K=c w \bar{w}$ which is a flat metric on $\mathbf{C}^{*}$. The Special Kähler structure is non-trivial however since $\nabla(d w)=0$ implies that the connection $\nabla$ in the coordinate $a$ is

$$
\nabla=\frac{d}{d a}+\frac{1}{2 a}
$$

and has a pole at $a=0$ with nontrivial holonomy. 
Remark: In general we observe that the complex structure on $\mathcal{B}^{\text {reg }}$ extends to $\mathcal{B}_{d} \subset \mathcal{B}$ and Proposition 2 shows that the Kähler potential extends, continously but not smoothly. It is the flat connection component of the Special Kähler structure which acquires a singularity, but this is typically a logarithmic singularity which induces a connection on the divisor. For the Gauss-Manin connection considered here the induced connection is presumably the flat connection for the Special Kähler structure on the divisor $\mathcal{B}_{1}$ where the spectral curve acquires a node.

\subsection{Fixed points of a $\mathrm{Z}_{n}$-action}

The last example admits a generalization to further cases where the metric is flat. Consider the action on $\mathcal{M}$ of tensoring $V$ with a line bundle $U$ such that $U^{n}$ is trivial, where $n=\operatorname{rk} V$ as usual. The fixed point set of this $\mathbf{Z}_{n}$-action is described in [4].

Firstly, having a fixed point means we have an isomorphism $\varphi: V \rightarrow V \otimes U$. This is a Higgs bundle where the twist is by $U$ instead of $K$ but the general theory tells us that $V$ is the direct image of a line bundle $L$ on the unramified cyclic $n$-fold cover $p: C \rightarrow \Sigma$ associated to $[U] \in H^{1}\left(\Sigma, \mathbf{Z}_{n}\right)$. Let $\sigma$ denote a generator of the $\mathbf{Z}_{n}$-action on $C$.

Since $p^{*} U$ is trivial, $p^{*} \varphi$ is an endomorphism of $p^{*} V$. To be a fixed point the Higgs field $\Phi$ must commute with $\varphi$ and so on $C$

$$
p^{*} \Phi=c_{0}+c_{1} p^{*} \varphi+\cdots+c_{n-1} p^{*} \varphi^{n-1}
$$

where $c_{i}$ are sections of $p^{*} K$. Then $\Phi=c_{0}+c_{1} \varphi+\cdots+c_{n-1} \varphi^{n-1}$ for sections $c_{k} \in H^{0}\left(\Sigma, K U^{-k}\right)$.

Since $p: C \rightarrow \Sigma$ is unramified, $p^{*} K \cong K_{C}$ and then the $c_{i}$ are sections of $K_{C}$ which transform as $\omega^{-i}$ under the action of $\sigma$, and an arbitrary 1-form on $C$ can be decomposed this way. The data is then just a holomorphic 1-form and a line bundle on $C$. This gives an integrable subsystem with a flat Special Kähler metric.

Since $\left(p^{*} \varphi\right)^{n}=1$ its eigenvalues are $1, \omega, \omega^{2}, \ldots$ where $\omega=e^{2 \pi i / n}$ and so the eigenvalues of $p^{*} \Phi$ are $c_{0}+c_{1}+\ldots+c_{n-1}, c_{0}+\omega c_{1}+\ldots+\omega^{n-1} c_{n-1}$, etc. Put another way, $\gamma=c_{0}+c_{1}+\ldots+c_{n-1}$ is a 1 -form on $C$ and the eigenvalues are $\gamma, \sigma^{*} \gamma,\left(\sigma^{2}\right)^{*} \gamma, \ldots$ Hence the spectral curve $S$ has equation

$$
\prod_{k=0}^{n-1}\left(x-\left(\sigma^{k}\right)^{*} \gamma\right)=0
$$

On $C$ we have $n$ single-valued roots of the characteristic polynomial so there is a map 
from $C$ to the spectral curve $S$. Both cover $\Sigma$ by taking eigenvalues of $\Phi$ so the map is generically one-to-one. Since $C$ is nonsingular it is the normalization of $S$.

If $\gamma$ has a simple zero at $c \in C$ then the other eigenvalues are 1-forms with zeros at $\sigma(c), \sigma^{2}(c), \ldots$. Then equation (8) shows that $S$ has an ordinary singularity of multiplicity $n$ over $p(c) \in \Sigma$. Since $C$ is an unramified $n$-fold covering of $\Sigma$, we have $2 g(C)-2=n(2 g-2)$ and $\gamma$ has $n(2 g-2)$ zeros. Thus the number of singularities of this type on $S$ is $(2 g-2)$. But the arithmetic genus of $S$ is $n^{2}(g-1)+1$ and

$$
n^{2}(g-1)+1-(2 g-2) \frac{n(n-1)}{2}=n(g-1)+1=g(C)
$$

so $S$ has no more singularities.

It follows that the singularity type for this subintegrable system consists of $n$ ordinary $n$-fold singularities.

The simplest case is the group $S L(2, \mathbf{C})$ where $U \in H^{1}\left(\Sigma, \mathbf{Z}_{2}\right)$ is a non-trivial line bundle of order 2. Then $H^{0}(\Sigma, K U)$ has dimension $g-1$ and a section $s$ defines the quadratic differential $q=s^{2}$. Then the formula (2) gives a Kähler potential

$$
K=\frac{1}{2} \int_{\Sigma} \sqrt{q \bar{q}}=\frac{1}{2} \int_{\Sigma} s \bar{s}
$$

This Hermitian expression in $s$ gives the flat metric on $H^{0}(\Sigma, K U) \backslash\{0\} / \pm 1$.

\subsection{Hyperkähler metrics}

Any Special Kähler manifold gives a semiflat hyperkähler metric on a manifold modelled on its cotangent bundle [6],[8]. The moduli space of Higgs bundles $\mathcal{M}$ has a natural $L^{2}$-metric which has been considered in more detail quite recently as in [10], [5]. In particular, over $\mathcal{B}^{\text {reg }}$ it is shown that the semiflat metric is a good approximation to the actual metric. It seems likely, then, that the formula here for the potential could provide an approximation on the lower dimensional loci $\mathcal{M}_{d}$.

In fact, in support of this suggestion, the fixed point set in Section 5.3 is a hyperkähler submanifold of the genuine metric on $\mathcal{M}$ and (because one can take the direct image of a flat connection in an unramified cover) the induced metric is also flat.

\section{Acknowledgements}

The author wishes to thank the EPSRC Programme Grant "Symmetries and Correspondences" and the Instituto de Ciencias Matemáticas, Madrid for support during 
the preparation of this work, and David Baraglia for useful discussions. This paper is based on a talk given at the conference "Between topology and quantum field theory" at the University of Texas in Austin in celebration of Dan Freed's 60th birthday.

\section{References}

[1] D.Baraglia, Classification of the automorphism and isometry groups of Higgs bundle moduli spaces, Proc. London Math. Soc. 112 (2016), 827-854.

[2] D.Baraglia \& Z.Huang, Special Kähler geometry of the Hitchin system and topological recursion, arXiv 1707.04975v2.

[3] A.Beauville, M.S.Narasimhan \& S.Ramanan, Spectral curves and the generalised theta divisor, J. Reine Angew. Math. 398 (1989), 169 -179.

[4] E.Franco, P.B.Gothen,A.Oliveira \& A.Peón-Nieto, Unramified covers and branes on the Hitchin system, arXiv 1802.05237v2.

[5] L.Fredrickson, Exponential decay for the asymptotic geometry of the Hitchin metric, arXiv 1810.01554v2.

[6] D.S.Freed, Special Kähler manifolds, Comm. Math. Phys. 203 (1999), 31 - 52.

[7] S.J.Gates, Superspace formulation of new nonlinear sigma models, J. Nucl. Phys. B 238 (1984), 349 - 366.

[8] N.J.Hitchin, The moduli space of complex Lagrangian submanifolds, Asian. J. Math. 3 (1999), 77 - 91.

[9] N.J.Hitchin, Critical loci for Higgs bundles, Comm. Math. Phys. 366 (2019), $841-864$.

[10] R.Mazzeo, J.Swoboda, H.Weiss \& F.Witt, Asymptotic geometry of the Hitchin metric, Comm. Math. Phys. 367 (2019), 151 - 191.

[11] R.J.Walker, "Algebraic curves," Princeton University Press, Princeton NJ (1950).

[12] B. de Wit \& A. van Proyen, Potentials and symmetries of general gauged $N=2$ supergravity Yang-Mills models, Nucl. Phys. B 245 (1984), 89 - 117. 